\title{
Modeling the mechanical wear of the contact elements of pantograph during high-speed movement
}

\author{
Victor Philippov ${ }^{1, *}$ and Alexandr Smerdin $^{1}$ \\ ${ }^{1}$ Omsk State Transport University, Railway power supply Department, 644046, Omsk, Marx av. 35, \\ Russia
}

\begin{abstract}
The use of contact elements with an extended service life is one of the most economical and least costly ways to ensure reliable, economical and environmentally friendly transmission of electricity to rolling stock. To evaluate the service life in OSTU, a methodology for conducting experimental studies of contact pairs of pantograph has been developed and successfully tested, involving bench tests for each pair of contact materials "contact insert - contact wire" in order to determine their optimal combination to reduce wear and increase service life. Assessment of the amount of wear and prediction of the life of the contact elements are made, including using mathematical modeling. The purpose of this article is to improve the mechanical component of the wear model of the contact elements of pantograph in high-speed conditions. The results of experimental studies and theoretical calculations of the mechanical wear of contact elements are presented. The analysis of the graphs allows us to conclude that it is possible to use an improved mathematical model for modeling the mechanical component of the wear process of the elements of contact pairs with a maximum error value of not more than $5 \%$.
\end{abstract}

\section{Introduction}

In accordance with the long-term development program of Russian Railways until 2025, approved by Decree of the Government of the Russian Federation of March 19, 2019 No. 466-r, it is planned to increase the level of economic connectivity of the territory of Russia by expanding and modernizing the railway infrastructure, developing multimodal transportation expanding the network of high-speed and high-speed transportation. However, the expansion of the high-speed traffic network involves not only the construction of new railways with the appropriate infrastructure, but also the modernization of the structures of individual nodes of the existing rolling stock, as well as the introduction of energy and resource-saving technologies to ensure the transportation of passengers and goods at speeds above $200 \mathrm{~km} / \mathrm{h}$. Nevertheless, a change in the design of a node (for example, a current collector) is directly associated with significant financial and time costs, while the use of contact elements with an extended service life, providing reliable, economical and

* Corresponding author: fvm-omgups@ mail.ru 
environmentally friendly transmission of electricity to rolling stock, is more economical and less expensive.

Increasing the service life of the collector element can be achieved, among other things, by reducing wear by correctly selecting the elements of the contact pair, both from the point of view of their tribocompatibility and the ability to ensure high-quality current collector. For this, it is necessary to take into account the physical properties of the materials of contact pairs, as well as factors specific to operating conditions.

A small number of specialists are engaged in the study of wear of contact elements and contact wires in the world. So, G. Bucca, A. Collina in their work [1] describe a method for predicting the wear of slip rings and contact wires, and H. Yang, B. Hu, Ya. Liu, X. Cui, G. Jiang in [2] consider the process of interaction of a contact pair under conditions of highspeed motion and significant current loads. However, in Russia these studies cannot be fully applied due to the specifics of climatic and weather conditions, as well as operating conditions.

\section{Materials and methods}

The parameters affecting the wear of the elements of contact pairs are shown in Fig. 1. Electricity is transmitted to the traction rolling stock by means of an electric sliding contact, while for high-speed movement the magnitude of the traction current flowing through a pair of "contact wire - contact element" can reach $3.5 \mathrm{kA}$. Such current values cause increased heating of the elements of the contact pairs of current collector devices, and its thermal effect significantly increases electrical and electromechanical wear [3].

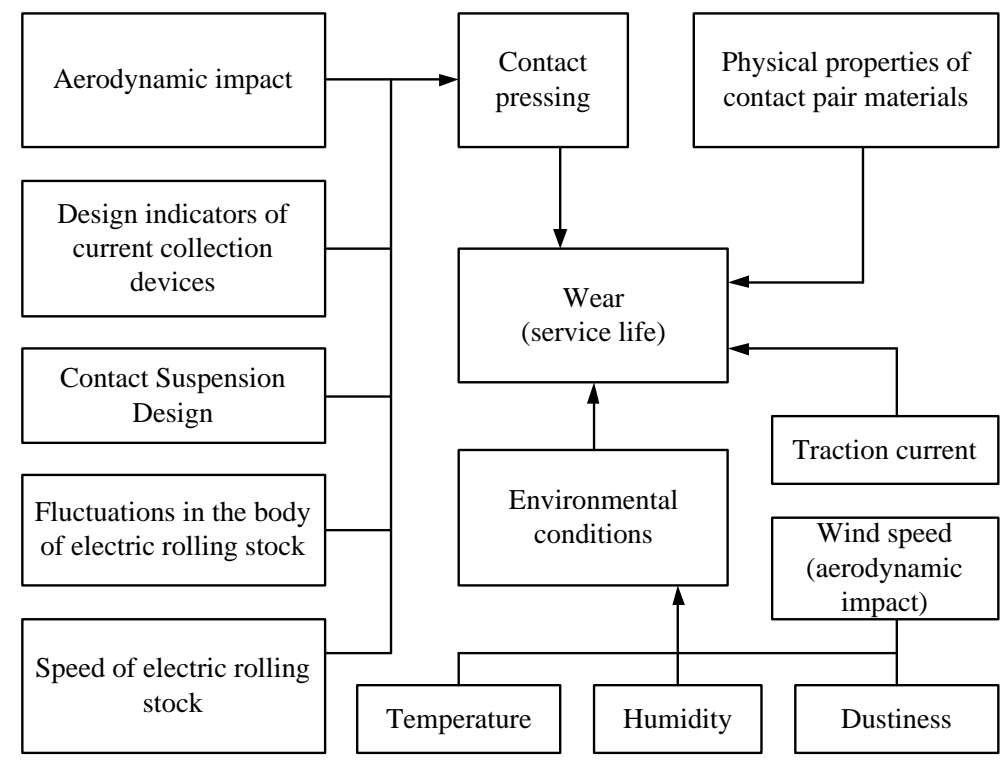

Fig. 1. Parameters affecting the wear of contact pair elements.

At Omsk State Transport University a program and methodology for conducting experimental studies of contact pairs of electric current collection devices has been developed and successfully tested.

The program involves bench testing for each pair of contact materials (tribosystems) "contact element - contact wire" in order to determine their optimal combination to reduce wear and increase resource and includes the following steps. 
1. Determination of tribocompatibility, scoring resistance and wear resistance of materials (studies from mechanical load in contact without electric current flowing). For this, the following parameters vary: contact pressing, dustiness and humidity of the ambient air, aerodynamic effect of the air flow, speed of movement.

2. The study of the wear of a pair of "contact element - contact wire" depending on the magnitude of the flowing current. To determine the characteristics of wear resistance, it is necessary to change the following parameters: contact pressure values, traction current values (or current density), environmental parameters and contact suspension at critical pressure values in the contact and speed. When implementing the indicated stage of the research methodology, it is necessary to perform it with alternating and direct current.

3. Assessment of the amount of wear and prediction of the resource of the elements of the contact pair using mathematical models.

The frictional interaction of the elements of the "contact element - contact wire" tribosystem is a nonlinear process that is determined by a large number of both internal (physicochemical properties of the friction pair materials) and external (dynamic loads, the presence of electric current in the contact, environmental parameters, etc.) interrelated factors.

To ensure the reliability of the data array obtained as a result of the tests, an algorithm for the implementation and processing of experimental data (Fig. 2) is applied, developed taking into account the provisions of the theory of experimental design.

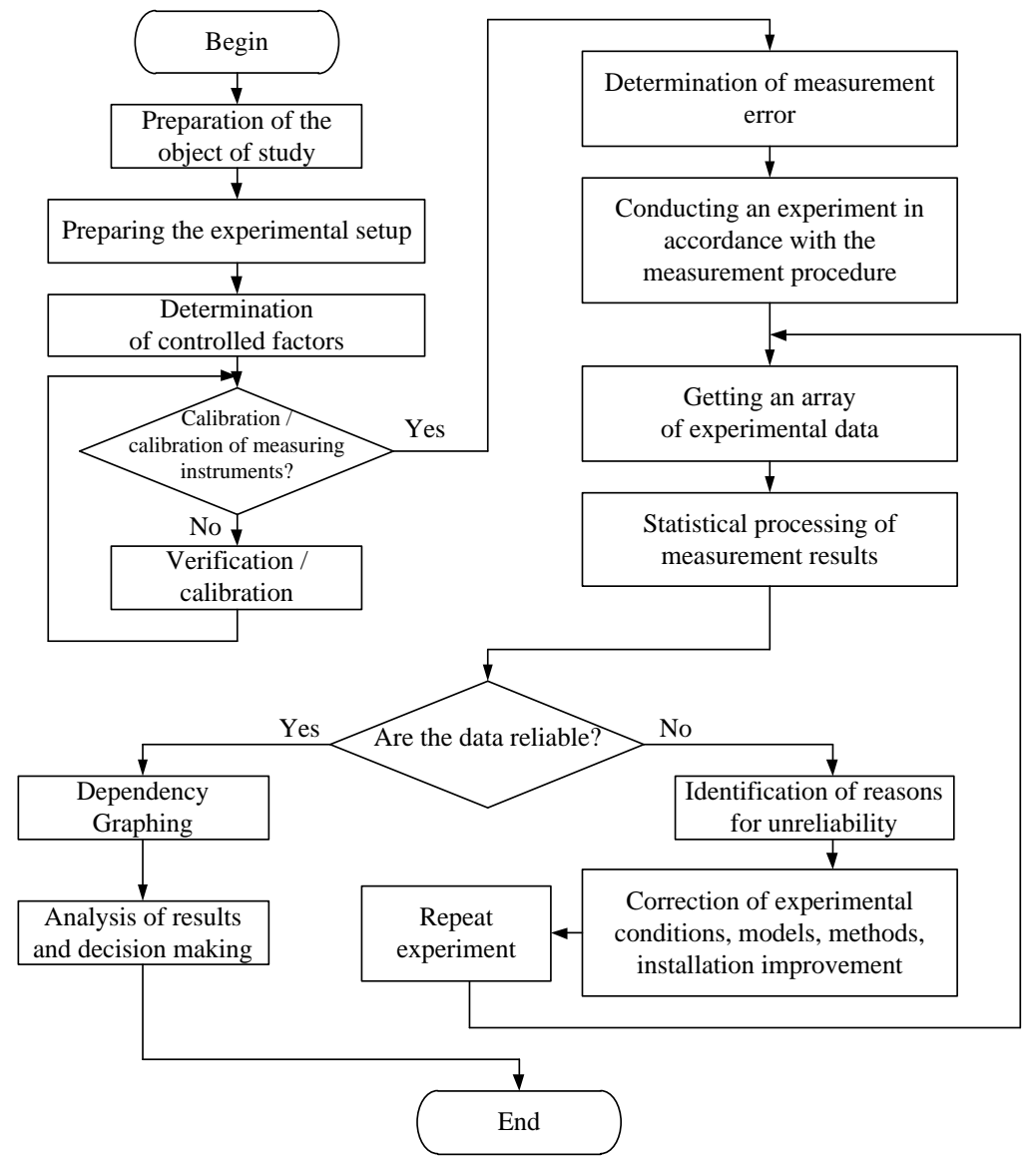

Fig. 2. Experiment Planning Algorithm. 
The results of experimental studies at high-speed movement depend on the parameters and characteristics of measuring instruments, the object of study, the quality of bench installations, as well as the human factor, the influence of which is manifested in the form of random errors and errors during the experiment [4]. Processing of the experimental results and prediction of wear and resource of the element of the contact pair of the current collector are carried out using mathematical models.

It should be noted that the mathematical model given in [3] takes into account the majority of those shown in Fig. 1 factors, however, its use for predicting wear under conditions of high-speed movement will not be sufficiently accurate due to the lack of consideration of the aerodynamic effects of air flow and the speed of movement of rolling stock on processes occurring in an electric sliding contact.

In this article, we consider the improvement of the mechanical component of the wear model for high-speed movement.

\section{Discussions}

In general terms, the basic functional relationship between the mechanical wear component $I_{\mathrm{m}}$ and model factors is described in [1] and is presented in the form:

$$
I_{\mathrm{m}}=\psi_{2}\left(P, v, t, r, \mathrm{Cu}, \mathrm{H}_{1} / \mathrm{H}_{2}, \lambda_{1}, \lambda_{2}, c_{1}, c_{2}\right),
$$

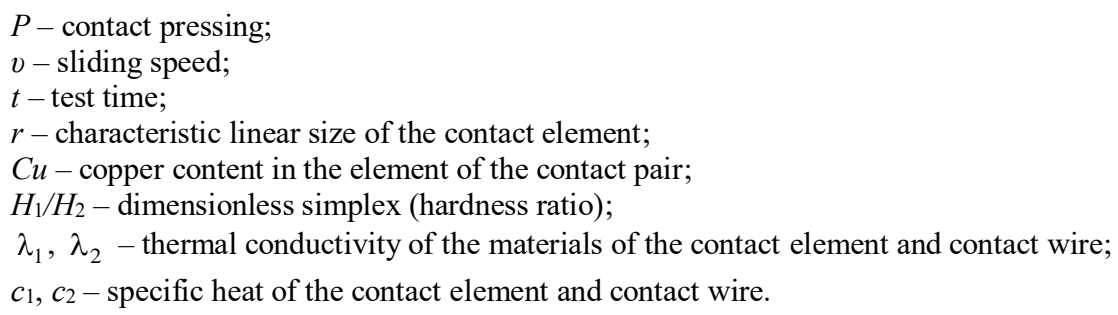

An initial analysis of the results of experimental studies in conditions of high-speed movement confirmed the need for introducing into the model criteria that take into account the state of the environment, the speed of aerodynamic effects, the influence of the temperature of the contact pair elements on the actual contact area [5] and their hardness, as well as a criterion that takes into account the effect of friction in sliding contact.

In equation (1), in accordance with the provisions of the theory of similarity, individual factors are combined into simplexes and complexes [4]. In this case, the following assumptions are made when forming the model:

1) side factors are combined into criteria (simplexes);

2) difficult to reproduce parameters are combined into complexes;

3 ) the calculation of approximate values of scale factors is carried out according to empirical formulas.

According to the $\pi$-theorem, the number of dimensionless combinations of complexes and simplexes is equal to the difference between the number of physical quantities and basic units of dimension. Thus, the following equation is obtained:

$$
I_{\mathrm{m}}(P)=a_{0} \cdot M e \cdot\left(\frac{P t c_{2}}{r^{2} \rho_{1} c_{1} \chi}\right)^{\alpha_{m}}\left(B i \frac{v t}{r}\right)^{\beta_{m}}(C u)^{\gamma_{m}}\left(\frac{H_{1}}{H_{2}}\right)^{\varepsilon_{m}}\left[\frac{\varphi_{0} \kappa_{0}}{\varphi_{1} \kappa_{1}}\left(0,1+v_{\mathrm{B}}\right)^{\frac{1}{2}}\right]^{\lambda_{m}},
$$

$a_{0}$ - functionality reflecting the influence of unaccounted factors on the process; 
$\alpha_{m}$ - functionality logarithmically dependent on the magnitude of contact pressing at a given time;

$\beta_{m}, \chi_{m}, \varepsilon_{m}, \lambda_{m}$ - experimentally determined coefficients;

$\operatorname{Ptc}_{2} /\left(r^{2} \rho_{1} c_{1} \chi\right)$ - complex (a measure of the ratio of the specific friction power to the ability of the contact wire to accumulate, and the contact element to transfer heat);

$c_{2}$ - specific heat of a contact wire;

$v t / r$ - ratio of the friction path to the surface coefficient of the contact element;

$M e \equiv \frac{T_{i} \lambda_{1}}{r f_{\mathrm{T}} p v}-$ Meyer criterion ( $p v-$ friction power (taking into account the distribution coefficient of heat fluxes), $f_{\mathrm{r}}-$ friction coefficient);

$B i=\frac{r \nabla T_{1}}{\Delta T_{1 \mathrm{~B}}}-$ Bio criterion;

$r \nabla T_{1}$ - temperature difference across the volume of the contact element;

$\Delta T_{1 \mathrm{~B}}=T_{1}-T_{\mathrm{B}}-$ temperature head (temperature difference between the material of the contact element and the environment);

$\frac{\varphi_{0} \kappa_{0}}{\varphi_{1} \kappa_{1}}\left(0,1+v_{\mathrm{B}}\right)^{\frac{1}{2}}-$ complex taking into account the influence of environmental factors $\left(\varphi_{0} / \varphi_{1}-\right.$ the ratio of the average value of relative humidity for five years to the value of relative humidity at the time of research, $\kappa_{0} / \kappa_{1}$ - the ratio of the average dust content of the environment over five years to the value of its dust content at the time of research, $v_{\mathrm{B}}$ - counter airflow aerodynamic impact on the contact element).

Nomograms and ranges of variation of coefficients and functionals for calculation by model (2) are given in [1].

Thermal processes in the elements of the contact pair of the current collector are mathematically described by a system of partial differential Poisson equations. The thermal state of the nth body is characterized by the differential heat balance equation:

$$
c_{n} \frac{d T_{n}}{d t}=\left(-\sum_{i=1}^{q(i \neq n)} \Lambda_{i n}\right) T_{n}+\sum_{i=1}^{q(i \neq n)}\left(\Lambda_{i n} T_{i}\right)+\sum \Delta U I_{n},
$$

$c_{n}$ - heat capacity of the $n$-th body;

$n$ - the number of bodies thermally related to the body $n$;

$\Lambda_{\text {in }}$ - heat transfer from the $i$-th body to the $n$-th body;

$\sum \Delta U I_{n}$ - power losses of the $n$-th body from the flow of electric current;

$t$ - time.

$$
c_{1}=\frac{1}{T_{10}}\left(\Lambda_{13}+\Lambda_{3 \mathrm{~B}}\right) \int T_{1} d t+\frac{1}{T_{10}} \int \sum\left(\Lambda_{13}+\Lambda_{3 \mathrm{~B}}\right) T_{3} d t+\frac{\Delta U I t}{T_{10}}
$$

$\Lambda_{\text {in }}=\alpha\left(T_{n}-T_{i}\right)$

$\alpha-$ heat transfer coefficient, $\alpha=\frac{m_{1}}{v}+m_{2} v_{\mathrm{B}}^{\frac{1}{2}}[3]$;

$T_{n}$ - temperature of the $n$-th body.; 
$T_{i}$ - temperature of the $i$-th body.;

$v_{\mathrm{B}}-$ oncoming air speed;

$v$ - relative velocity of the contact wire and contact element in the horizontal plane perpendicular to the axis of the path;

$m_{1}, m_{2}$ - experimentally determined coefficients.

In equation (4) the following notation is adopted: 1 - contact element; 3 - skid of the current collector; B - air flow.

It should be noted that at speeds of up to $60 \mathrm{~km} / \mathrm{h}$ the discrepancy between the experimental and calculated data does not exceed 5\%, and at speeds above $150 \mathrm{~km} / \mathrm{h}$ the error becomes more than $10 \%$.

In Fig. 3 shows the graphs of the mechanical wear of the contact elements obtained experimentally and as a result of calculation according to the existing (Fig. 3, a) and advanced (Fig. 3, b) mathematical models (equations (2) - (4)). The graphs were obtained under normal environmental conditions (in accordance with Russian State Standard 8.050-73 "State system for ensuring the uniformity of measurements. Normal conditions for linear and angular measurements") and an air flow rate of $50 \mathrm{~m} / \mathrm{s}$.

In Fig. 3, the notation is accepted: 1 - experimental results; 2 - calculation results.
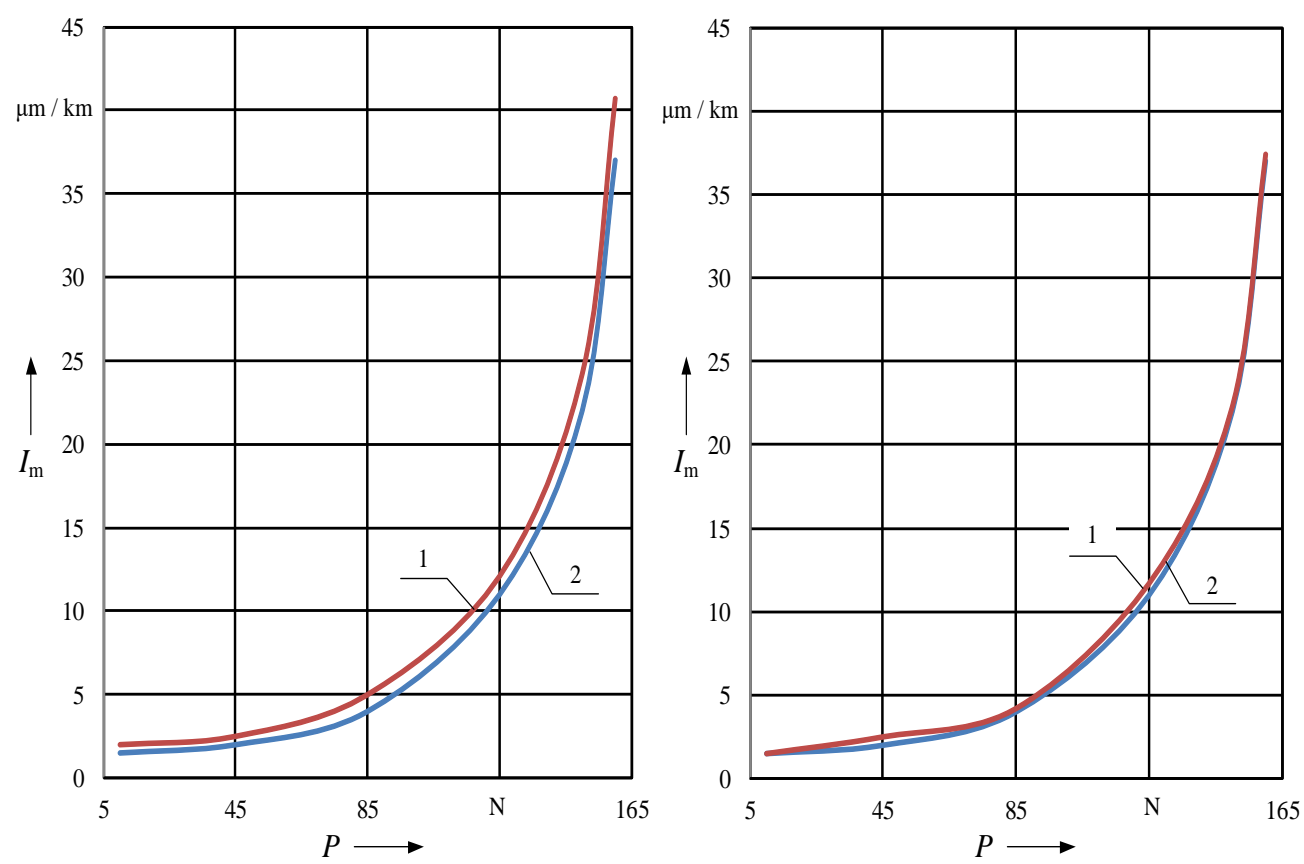

Fig. 3. Wear rate of a graphite contact element according to the existing (a) and advanced (b) models.

\section{Conclusions}

The analysis of the graphs in Fig. 3 allows us to conclude that the calculation accuracy is increased according to the mathematical model (2) by taking into account the influence of the aerodynamic effects of the air flow and the speed of the rolling stock to simulate the 
mechanical component of the wear of the elements of contact pairs at high speeds (the error decreased from $10 \%$ to $5 \%$ ).

\section{References}

1. G. Bucca, A. Collina. A procedure for the wear prediction of collector strip and contact wire in pantograph-catenary system. Wear 266 (2009). https://doi.org/10.1016/j.wear.2008.05.006

2. H. Yang, B. Hu, Ya. Liu, X. Cui, G. Jiang. Influence of reciprocating distance on the delamination wear of the carbon strip in pantograph-catenary system at high slidingspeed with strong electrical current. Engineering Failure Analysis 104 (2019). https://doi.org/10.1016/j.engfailanal.2019.06.060

3. O. Sidorov, V. Philippov, S. Stupakov. Studies of the electromechanical wear of contact pairs in the current collection devices of electric transports. J. Frict. Wear 36 (2015). https://doi.org/10.3103/S1068366615050128

4. H. Schenck. Theories of Engineering Experimentation (1967). https://doi.org/10.1115/1.3641806

5. H. Biesenack, F. Pintscher. Kontakt zwischen Fahrdraht und SchleifleisteAusgangspunkte zur Bestimmung des elektrischen Verschleißes. Elektrische Bahnen 3 (2005)

6. Ilinykh, A. Manakov, A. Abramov, S. Kolarzh, MATEC Web of Conferences 216, 03004 (2018). DOI: 10.1051/matecconf/201821603004

7. A.L. Manakov, A.D. Abramov, A.S. Ilinykh, M.S. Galay, J.S. Sidorov, Journal of Physics: Conference Series 1050(1), 012051 (2018). DOI: 10.1088/17426596/1050/1/012051

8. L. Lazarević, D. Vučković, Z. Popović, Proceedings of the Institution of Mechanical Engineers, Part F: Journal of Rail and Rapid Transit 230(8), 1828-1841 (2016).

9. M. Vilotijević, M. Vukićević, L. Lazarević, Z. Popović, Tehnički vjesnik 25(2), 516523 (2018).

10. L. Lazarević, D. Vučković, M. Vilotijević, Z. Popović, Structural Health Monitoring 18(3), 792-805 (2019). 